\title{
Evaluación de metodología flipped classroom: primera experiencia
}

\section{Flipped Classroom Assessment Method: first experience}

\author{
Angélica Rosa Opazo Faundez¹, José Miguel Acuña Bastias², María Priscilla Rojas Polanco ${ }^{3}$ \\ ${ }^{1}$ Universidad Santo Tomás, Chile (angelicaopazofa@ santotomas.cl) \\ ${ }^{2}$ Universidad Santo Tomás, Chile (joseacunaba@ santotomas.cl) \\ ${ }^{3}$ Departamento Desarrollo Curricular, Universidad Santo Tomás, Chile (mprojas@ santotomas.cl)
}

Recibido el 14 de agosto de 2016; revisado el 15 de septiembre de 2016; aceptado el 17 de septiembre de 2016; publicado el 2 de diciembre de 2016

\section{RESUMEN:}

Flipped classroom (FC) es una metodología de enseñanza aprendizaje que aplica las tecnologías de la información y comunicación (TIC) e invierte las actividades que tradicionalmente se realizan en clases, con las actividades que los estudiantes realizan fuera del aula. El objetivo de este trabajo fue implementar y evaluar la metodología flipped classroom en una unidad de la asignatura de Hematología Clínica de la carrera Tecnología Médica, Universidad Santo Tomás, Santiago de Chile. La muestra de este estudio estuvo constituida por 28 estudiantes matriculados en la asignatura de Hematología Clínica el año 2015. Se realizó encuesta de satisfacción a los estudiantes con respecto a la metodología FC y se evaluó el impacto de la metodología en los resultados de aprendizaje de los estudiantes.

Según la encuesta aplicada, el $65 \%$ de los estudiantes prefieren FC en vez de las clases tradicionales y el $60 \%$ dice que aprende más cuando se utiliza FC. En la calificación de los resultados de aprendizaje abordados con esta metodología se observó un aumento de 5\% de las calificaciones comparado con los estudiantes del año anterior. Sin embargo, la comparación global de notas no es estadísticamente significativa $(p>0,5)$, pero cuando se compara el número de estudiantes que obtienen más o menos de nota 4,0 en la prueba la diferencia es estadísticamente significativa $(p<0,5)$. En el análisis de los resultados de aprendizaje evaluados en este estudio se observa que mejora el rendimiento académico de la mayoría de estudiantes.
La metodología flipped classroom es evaluada satisfactoriamente por los estudiantes y podría mejorar el rendimiento académico de los estudiantes.

PALABRAS CLAVE: FLIPPED CLASSROOM, APRENDIZAJE ACTIVO, APRENDIZAJE PARTICIPATIVO.

\section{ABSTRACT:}

Flipped Classroom (FC) is a teaching learning methodology that applied the Information and Communication Technology, and where the traditional activities perform by the students were invert with the activities that they do out of classroom. The aim of this work was to implement and evaluate the Flipped Classroom methodology in a unit of the Clinical Haematology course of Medical Technology career in Universidad Santo Tomas, Santiago de Chile.

The sample of this study was constituted by 28 students registered in Clinical Haematology course in 2015. To evaluate the FC methodology, we applied a satisfaction survey to the students and the impact of the methodology was evaluate respect of learning of the students.

According to results obtained in the survey, $65 \%$ of the students preferred FC instead of the traditional classes and $60 \%$ said that they learned more when FC was used. Respect to the qualification of learning tackled with this methodology, there was observed an increase of $5 \%$ compared with the students of the previous year, nevertheless the comparison of global notes are not statistically significant $(p>0,5)$. In the 
comparison, the number of students whom obtained more of a 4,0 in their qualification in the test respect at 2015, the difference was statistically significant $(p<0,5)$. In the analysis of the learning evaluated in this study, it was observed that it improves the academic yield of most of students.

The FC methodology was satisfactory evaluated by the students and it could be a strategic to improved their academic performance in this or other classes

\section{KEYWORDS: FLIPPED CLASSROOM, ACTIVE} LEARNING, PARTICIPATORY LEARNING.

\section{INTRODUCCION}

\subsubsection{Planteamiento del problema}

De acuerdo al cambio de paradigma en educación y consecuente surgimiento del modelo educativo centrado en el estudiante y en el aprendizaje, ha provocado que los sistemas educativos deban hacer una renovación de los contenidos, métodos de enseñanza y de aprendizaje, prácticas y medios de transmisión de los saberes, con la finalidad de implementar métodos educativos innovadores que faciliten la creación del pensamiento crítico y creatividad en las personas de acuerdo a los requerimientos de la actual sociedad del conocimiento y de la información (Unesco 2005, 2006). Por lo tanto, la incorporación de metodologías de enseñanza y aprendizaje innovadoras ofrece una oportunidad para dar solución a los nuevos requerimientos de los planes de estudio.

Por otro lado, existen estudios que evidencian que la clase magistral tradicional no permite el desarrollo de competencias transversales esperadas para un egresado de la educación superior en el siglo XXI. Es por eso que al adaptar nuevos currículos a competencias o basados en competencias se hace indispensable innovar e incorporar al aula nuevas metodologías al proceso de enseñanza aprendizaje, que permitan diversificar el proceso, centrarse en el aprendizaje del estudiante, optimizar los tiempos de encuentro del docente con el estudiante, centrarse en niveles de aprendizajes superiores según la taxonomía de Bloom, focalizándose en el aprendizaje significativo, pensamiento crítico, y no solo en la memorización y repetición de contenidos (Arias, 2007).

Un segundo factor es que principalmente en la última década a nivel mundial y nacional se ha observado el fenómeno de masificación de la educación superior y diversificación del perfil del alumnado que ingresa a este. Estos estudiantes son principalmente pertenecientes a la llamada generación Millennials, personas nacidas entre el año 1982 al 2004, y como los describe Phillips (2014), son personas prefieren experiencias de aprendizaje interactivo, experimental y son dependientes de la tecnología. Estos estudiantes han comenzado a ingresar de manera creciente a la educación superior desde el año 2000 y se ha demostrado que requieren y necesitan enfoques diferentes para el aprendizaje. Son descritos como inteligentes, ambiciosos, con diversidad cultural, están siempre conectados a la tecnología, esperan respuestas inmediatas, son amantes de la cultura digital, esperan obtener lo que necesitan y para ellos es importante el buen rendimiento, las calificaciones y el éxito académico (Phillips, 2014; Phitayakorn 2015). Por lo tanto, las instituciones de educación deben conocer y entender estas necesidades de los estudiantes que arriban a la educación superior, con el fin de brindar una formación efectiva y optimizar los métodos de enseñanza acorde a las necesidades de aprendizaje de los estudiantes y que abarquen ampliamente el estilo de aprendizaje de estos (Fukushi, 2010).

Las oportunidades que ofrece la tecnología, permite generar instancias de aplicación de nuevos métodos de enseñanza-aprendizaje que resulten motivantes y desafiantes para los estudiantes. De esta manera, la penetración de las tecnologías significa cambios a nivel institucional y en las formas de realizar la enseñanza.

Es por esto que investigaciones a nivel mundial han demostrado que las TIC pueden conducir a mejorar el aprendizaje de los estudiantes y la efectividad de los métodos de enseñanza (Padilla, 2007), además de hacer de las clases una experiencia novedosa y atractiva. Uno de los métodos innovadores que a nivel internacional se ha estado utilizando últimamente es el FC, también conocido como aula invertida.

Por ello, se plantea este trabajo de innovación académica con el objetivo de incluir en la enseñanza de Tecnología Médica, metodologías de aprendizaje activo, fomentando la participación de los estudiantes en el aprendizaje autónomo. En este trabajo se aplica la metodología FC para el desarrollo de la III Unidad de la asignatura de Hematología clínica 1-2015, perteneciente al VII semestre del plan de estudio número 5 de la carrera de Tecnología Médica de Universidad Santo Tomás, sede Santiago de Chile, como reemplazo de la metodología centrada en el docente, propia de clases tradicionales. 


\subsection{Fundamentación teórica.}

FC o aula invertida es un método de enseñanzaaprendizaje que invierte la forma en la cual se realiza la enseñanza tradicional, es decir, invierte las actividades que se realizan en el aula, con las que el estudiante realiza fuera de esta. (Bergmann, 2012; Berrett, 2012; Gilboy, 2015). Las actividades que el docente tradicionalmente realiza en la clase (presentaciones o exposiciones) los estudiantes las ven antes de asistir a la clase presencial, mediante vídeos que el profesor dispone online y, de esta manera, el estudiante realiza individualmente el aprendizaje de menor nivel cognitivo según la pirámide de Miller, como aprendizaje de conceptos, definiciones, etc. (Healey, 2000). El tiempo de clases se dedica actividades de aprendizaje activo grupales o individuales en las cuales se requiere que el estudiante aplique y analice los conceptos a un nivel más alto de cognición, resuelva dudas sobre aspectos más difíciles de los contenidos del curso, con la ayuda de un docente facilitador, quien da retroalimentación inmediata y realiza una evaluación rápida de las ideas claves de la clase (Baepler, 2014; Bergmann, 2012; Berrett, 2012; Gilboy, 2015; Tourón, Santiago y Díez 2014).

La palabra FLIP está dada por los términos anglosajones de Flexible environment, Learning culture, Intentional content y Professional educator (Yarbro, 2014). El aprendizaje flexible está dado por el acceso libre a consulta del material de estudio que se publica online y que está disponible para que cada alumno consulte según sus necesidades individuales; además por estar enmarcado en una cultura de aprendizaje, en la cual el estudiante aprende antes de las clases y, fundamentalmente, en las clases en las cuales el docente planifica actividades de trabajo que tienen por objetivo la exploración de los temas con mayor profundidad y con la oportunidad de crear experiencias de aprendizaje de mayor riqueza; dicha planificación está enfocada en contenidos intencionalmente dirigidos para lograr la construcción del conocimiento; todo esto creado y dirigido por un docente facilitador profesional que vela por el cumplimiento de los objetivos de la metodología y del programa de estudio (Yarbro, 2014).

Una de las ventajas más destacadas de esta metodología es que el aprendizaje y la responsabilidad del su logro recae en el estudiante, haciendo uso de las TIC y de metodologías activas y participativas. Todo esto permite desarrollar y potenciar el pensamiento crítico, aprendizaje autónomo, capacidad de análisis, síntesis y evaluación de manera conjunta con las capacidades de trabajo en equipo, pro-actividad, gestión del tiempo, creatividad, competencias específicas en lo disciplinar, capacidad de adaptación y de respuestas a las demandas cambiantes del ejercicio profesional y a las competencias transversales necesarias para los profesionales del siglo XXI (Pozo y Pérez, 2011). Esto es avalado por diversos estudios (Berrett, 2012; Das y Sakar, 2015; Gilboy, 2015; Hughes, 2012; Kachka, 2012; Khan Academy, 2010; Zappe, 2009) que plantean que este método ayuda y facilita el aprendizaje, incentiva y responsabiliza a los estudiantes a ser aprendices activos en su propio aprendizaje, en concordancia al cambio de paradigma en educación.

Actualmente la implementación del método FC se facilita debido a disponibilidad y masificación del uso de las TIC, a la disponibilidad de vídeos online y el creciente acceso de los estudiantes a la tecnología. La incorporación de la tecnología al aula es beneficiosa, debido a que permite incorporar los contenidos clave de la asignatura por medio de vídeos, material de lectura u otros medios, a los cuales el estudiante pueden acceder a su propio ritmo de aprendizaje y en un fácil acceso (Whillier y Lystad, 2015).

La mayoría de estas investigaciones sobre FC emplean actividades de aprendizaje interactivas basadas en grupos de trabajo dentro el aula, citando las teorías de aprendizaje centradas en el estudiante (Bishop y Verleger, 2013), enmarcada en la integración del constructivismo y cognitivismo (Bergmann, 2013; Yestrebsky, 2015). Varios estudios realizados en diversas disciplinas y niveles formativos, tanto en educación secundaria en áreas de las matemáticas (Kachka, 2012; Khan Academy, 2010), aplicaciones informáticas (Johnson y Renner, 2012) y en la educación superior (Berrett, 2012; Das y Sakar, 2015; Gilboy, 2015; Hughes, 2012; Zappe, 2009), han demostrado que el uso de este método de clase invertida podría conducir a mejores resultados de aprendizaje y aumentar el desarrollo de estrategias y prácticas en la resolución de problemas, pero no existe suficiente evidencia científica para concluir si este método mejoraría el rendimiento académico. Aunque existen trabajos publicados sobre la implementación de la metodología no hay suficiente evidencia que evalúe el impacto real de esta metodología en los resultados de aprendizaje de los estudiantes.

El objetivo general de este estudio es implementar la metodología de FC en la III unidad de la asignatura de Hematología Clínica de la carrera Tecnología Médica de la Universidad Santo Tomás Santiago y evaluar la percepción de ella. 
Como objetivos específicos se plantean:

- Diseñar planificación de clases de la III unidad de la asignatura de Hematología Clínica; incorporando la metodología de FC como reemplazo de las clases de cátedra tradicionales.

- Aplicar la metodología de FC basada en experiencias internacionales, en la III unidad de la asignatura de Hematología Clínica, siguiendo planificación propuesta.

- Determinar el nivel de satisfacción de los estudiantes participantes del estudio respecto a la metodología de FC vivenciada.

- Evaluar el nivel de logro de los resultados de aprendizajes de los estudiantes cuando se aplica el método de FC en comparación los resultados del año anterior de la III unidad de la asignatura en la cual se realizaron clases tradicionales.

\section{MATERIAL Y MÉTODO}

\subsection{Diseño}

Por las características del estudio se trata de un diseño de investigación evaluativa. En este estudio se aplicará la metodología de FC a un grupo de estudiantes; posteriormente, se evaluará la percepción de los estudiantes sobre el uso de esta metodología y el impacto en los resultados de aprendizajes.

\subsection{Muestra}

La muestra está formada por 28 estudiantes, que corresponden a todos los estudiantes inscritos en la asignatura de Hematología Clínica en el primer semestre académico del año 2015 (1-2015) de la carrera de Tecnología Médica de la Universidad Santo Tomás sede Santiago. Se utilizó como grupo de comparación a 56 estudiantes que cursaron la misma asignatura el año anterior (1-2014) cuyas clases se realizaron con metodología tradicional. El detalle demográfico de los grupos se observa en la tabla 1.

Tabla 1. Detalle demográfico del grupo del estudio

\begin{tabular}{|c|c|c|c|c|}
\hline Grupos & $\begin{array}{c}\text { Hombres } \\
\mathrm{N} \\
(\%)\end{array}$ & $\begin{array}{c}\text { Mujeres } \\
\mathrm{N} \\
(\%)\end{array}$ & Total & $\begin{array}{c}\text { Edad } \\
\text { años } \\
(X \\
\pm D E) \\
\end{array}$ \\
\hline $\begin{array}{c}\text { Grupo } \\
\text { muestra } \\
(1-2015)\end{array}$ & $\begin{array}{c}11 \\
(39,3 \%)\end{array}$ & $\begin{array}{c}17 \\
(60,6 \%)\end{array}$ & 28 & $\begin{array}{c}24,3 \pm \\
2,5\end{array}$ \\
\hline $\begin{array}{c}\text { Grupo } \\
\text { comparación } \\
(1-2014)\end{array}$ & $\begin{array}{c}21 \\
(37,5 \%)\end{array}$ & $\begin{array}{c}35 \\
(62,5 \%)\end{array}$ & 56 & $\begin{array}{l}23,6 \\
\pm 2,1\end{array}$ \\
\hline
\end{tabular}

\subsection{Método}

Se seleccionaron las clases correspondientes de la III unidad de la asignatura de Hematología Clínica, se realizó la planificación metodológica que consistió en planificar actividades para la etapa previa a las clases, de clases y post clase y la metodología se aplicó y desarrolló en 4 semanas, comprendidas entre el 26 de mayo al 20 de junio de 2015, con un total de 20 horas pedagógicas ( 2 sesiones de 2 horas pedagógicas cada una).

Las actividades realizadas durante la implementación de la metodología fueron:

Actividades previas a la clase: el contenido de las clases fue presentado a los estudiantes en la plataforma Moodle de Intranet Universidad Santo Tomás, en la sección de la asignatura de Hematología Clínica TME-079. El material estaba formado por vídeos de corta duración (15 minutos) que hacían una explicación de los contenidos elementales de la clase. Se utilizaron vídeos de Khan Academy, YouTube previamente seleccionados por el profesor, o de elaboración propia en Camtasia Studio, presentación en PowerPoint o una lectura de texto. El material estaba disponible en intranet con una semana de anticipación y era de libre acceso. El total de las actividades no sobrepasaba los 20 minutos de duración.

Actividades en clases: la clase comenzó con una evaluación formativa del tema tratado en las actividades previas a la clase, discusión breve del contenido mediante preguntas y respuestas utilizando clickers y/o breve exposición del profesor. Posteriormente la clase se dedicó a realizar trabajo activo en grupos de 3 a 5 integrantes, que contemplaban resolución de casos clínicos, discusión en grupo, etc.; actividades planificadas según los resultados de aprendizajes de la unidad. Los estudiantes accedían a información mediante smartphone, tableta o PC, libros de texto, etc.

Actividades post clases: los estudiantes debían profundizar los temas tratados antes y durante la clase y complementar con información adicional (guías clínicas nacionales de patologías hematológicas y bibliografía básica del curso). Además, se revisaba el trabajo elaborado por los estudiantes, se realizaba la retroalimentación y calificación formativa del mismo.

\subsection{Instrumentos de recopilación de datos}

- Encuesta de satisfacción. Para evaluar el nivel de satisfacción de la metodología FC se aplicó una encuesta de satisfacción. Se utilizó el cuestionario de Gilboy (2015) que consiste en un instrumento de respuesta 
anónima compuesto de 5 ítems, en los cuales las opciones de respuestas eran Muy de acuerdo, De acuerdo, Neutral, En desacuerdo o Muy en desacuerdo.

- Evaluaciones de conocimientos y productos durante el desarrollo de la unidad III. Este ítem contempló una evaluación que se realizaba al inicio de las clases, que consistía en una pregunta de desarrollo que evaluaba el contenido mínimo a abordar en la clase y que los estudiantes debían preparar mediante las actividades previas. También se ponderan en este ítem las evaluaciones del producto generado en las clases (trabajos realizados en grupo).

- Evaluación de conocimiento de la unidad III. Se realizó mediante una prueba de conocimientos de 50 puntos que contempló preguntas de selección múltiple y de desarrollo. Se calificó en escala de notas de 1 a 7 con exigencia del 60\%. La prueba estaba formada en un $48 \%$ de preguntas de conocimientos puros, un $24 \%$ de preguntas de comprensión y $28 \%$ de preguntas de análisis, aplicación o evaluación.

\subsection{Análisis de datos}

- Se analizaron y tabularon las respuestas de la encuesta de satisfacción.

- Análisis de logro de los resultado de aprendizaje de la unidad intervenida: en base a la tabla de especificaciones creada para la confección del instrumento evaluativo, se clasificaron las preguntas según el grado de complejidad, de acuerdo a la taxonomía de Bloom revisada de Anderson y Krathwohl, 2014) y clasificación utilizada en Universidad Santo Tomás (Riveros, 2013) y de acuerdo al juicio de expertos de la asignatura, como:

- Preguntas de conceptos: nombrar, definir, identificar, etc.

- Preguntas de comprensión. relacionar, ejemplificar, diferenciar, etc.

- Preguntas de análisis: explicación, aplicación, análisis, evaluación, etc.

Se calcularon porcentajes de las preguntas correctas e incorrectas según cada aprendizaje.

- Evaluación prueba de conocimientos de la unidad $\mathrm{y}$ evaluaciones de producto $\mathrm{y}$ conocimientos durante el desarrollo de la unidad III. Se realizó un estudio de distribución de notas y se compararon las calificaciones obtenidas de los estudiantes 12015, con las calificaciones obtenidas por los estudiantes del año anterior (1-2014). Se calculó media, error estándar y la prueba estadística no paramétrica de Mann-Whitney con un nivel se significancia $\mathrm{p}<0.05$.

Adicionalmente se realizó un análisis de las calificaciones obtenidas en la prueba de conocimientos de la III Unidad del curso entre los estudiantes del año 1-2015 y del 1-2014. Se comparó aprobación (estudiantes que obtienen una nota igual o mayor a 4.0) y reprobación (estudiantes que obtienen una nota menor o igual a 3,9). Se utilizó la prueba estadística Chi cuadrado (nivel de significancia $\mathrm{p}<0.05)$.

\section{RESULTADOS}

\subsection{Resultados de la implementación de FC}

Se implementó la metodología de FC en la III Unidad de la asignatura de Hematología Clínica en las fechas propuestas. Se diseñaron las planificaciones de clases para las sesiones de acuerdo a los contenidos del programa de Hematología Clínica TME-079, 1-2015 (tabla 2) y se publicó el material interactivo en el aula virtual de la asignatura en la página de intranet Moodle de Universidad Santo Tomás.

Tabla 2. Actividades Realizadas con FC

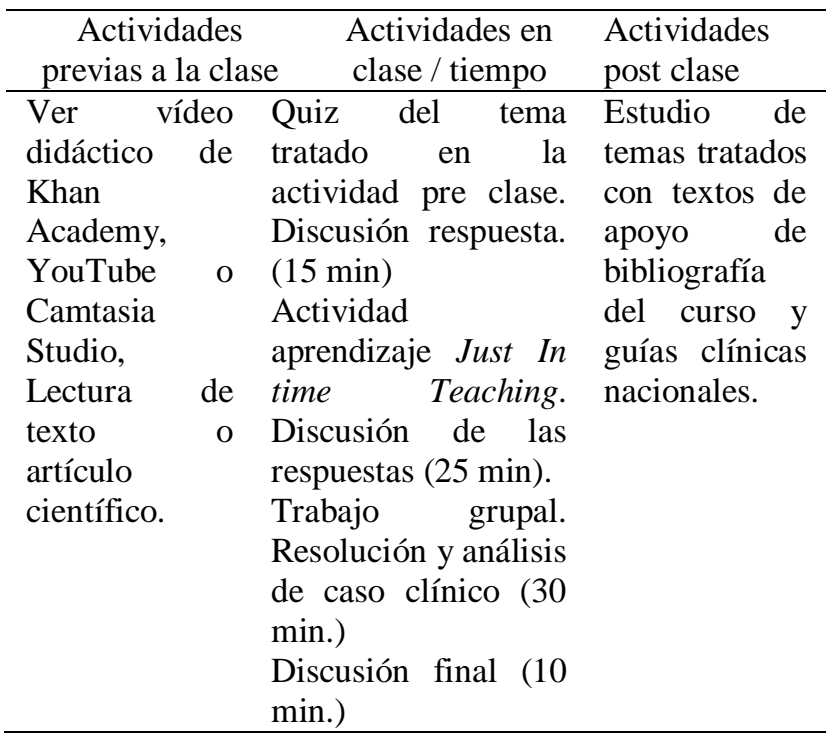

\subsection{Resultados de la encuesta de satisfacción}

A los 28 estudiantes seleccionados pertenecientes a la cohorte 2015 se les aplicó la encuesta de satisfacción. Se obtuvieron 26 encuestas contestadas (participación del 92,9\% de los estudiantes). 
Las respuestas a cada pregunta del cuestionario se encuentran graficadas en la tabla 3.

Tabla 3. Resultados encuesta de satisfacción

\begin{tabular}{|c|c|c|c|c|c|}
\hline Ítems & $\begin{array}{c}1 \\
\mathrm{~N}^{\circ}(\%)\end{array}$ & $\mathrm{N}^{\circ}(\%)$ & $\begin{array}{c}3 \\
\mathrm{~N}^{\circ}(\%)\end{array}$ & $\begin{array}{c}4 \\
\mathrm{~N}^{\circ}(\%)\end{array}$ & $\begin{array}{c}5 \\
\mathrm{~N}^{\circ}(\%)\end{array}$ \\
\hline $\begin{array}{l}\text { Ítem } 1 . \text { Me gustó } \mathrm{l}_{\mathrm{c}} \\
\text { una clase tradiciona }\end{array}$ & $\begin{array}{c}4 \\
(15,3)\end{array}$ & $\begin{array}{c}13 \\
(50,0)\end{array}$ & $\begin{array}{c}6 \\
(23,1)\end{array}$ & $\begin{array}{c}2 \\
(7,7)\end{array}$ & $\begin{array}{c}1 \\
(3,8)\end{array}$ \\
\hline $\begin{array}{l}\text { Ítem 2. Prefiero tener la clase tradicional (conferencia) del } \\
\text { profesor en vez de realizar trabajos activos y grupales en clases } \\
\text { como los que se llevaron a cabo con metodología FC. }\end{array}$ & $\begin{array}{c}0 \\
(0,0)\end{array}$ & $\begin{array}{c}4 \\
(15,4)\end{array}$ & $\begin{array}{c}9 \\
(35,0)\end{array}$ & $\begin{array}{c}11 \\
(42,3)\end{array}$ & $\begin{array}{c}2 \\
(7,7)\end{array}$ \\
\hline $\begin{array}{l}\text { Ítem 3. El uso de vídeos me permite aprender el material de } \\
\text { estudio más eficazmente que hacer las lecturas en solitario. }\end{array}$ & $\begin{array}{c}4 \\
(15,4)\end{array}$ & $\begin{array}{c}13 \\
(50,0)\end{array}$ & $\begin{array}{c}6 \\
(23,0) \\
\end{array}$ & $\begin{array}{c}2 \\
(7,7)\end{array}$ & $\begin{array}{c}1 \\
(3,8)\end{array}$ \\
\hline $\begin{array}{l}\text { Ítem 4. Yo aprendí más cuando utilicé el método de aprendizaje } \\
\text { FC (vídeos, lecturas cortas y actividades de aprendizaje activo en } \\
\text { clase) en comparación con el método tradicional (exposición del } \\
\text { profesor). }\end{array}$ & $\begin{array}{c}1 \\
(3,8)\end{array}$ & $\begin{array}{c}14 \\
(53,8)\end{array}$ & $\begin{array}{c}7 \\
(26,9)\end{array}$ & $\begin{array}{c}4 \\
(15,4)\end{array}$ & $\begin{array}{c}0 \\
(0,0)\end{array}$ \\
\hline $\begin{array}{l}\text { İtem 5. Me sentía desconectado sin un profesor presente durante } \\
\text { los vídeos o actividades virtuales. }\end{array}$ & $\begin{array}{c}2 \\
(7,7)\end{array}$ & $\begin{array}{c}3 \\
(11,5)\end{array}$ & $\begin{array}{c}6 \\
(23,0)\end{array}$ & $\begin{array}{c}11 \\
(42,3)\end{array}$ & $\begin{array}{c}4 \\
(15,4)\end{array}$ \\
\hline
\end{tabular}

1: Fuertemente de acuerdo; 2: De acuerdo; 3: Neutral; 4: En desacuerdo; 5: Fuertemente en desacuerdo

\subsection{Resultado de aprendizaje prueba de conocimientos.}

A los 28 estudiantes que participaron en este estudio se les aplicó la prueba de conocimiento y se obtuvo una nota promedio del curso de 4,3 (E.S=0,8). La distribución de porcentajes de preguntas correctas e incorrectas de cada tipo de pregunta del instrumento de evaluación se observa en la figura 1.

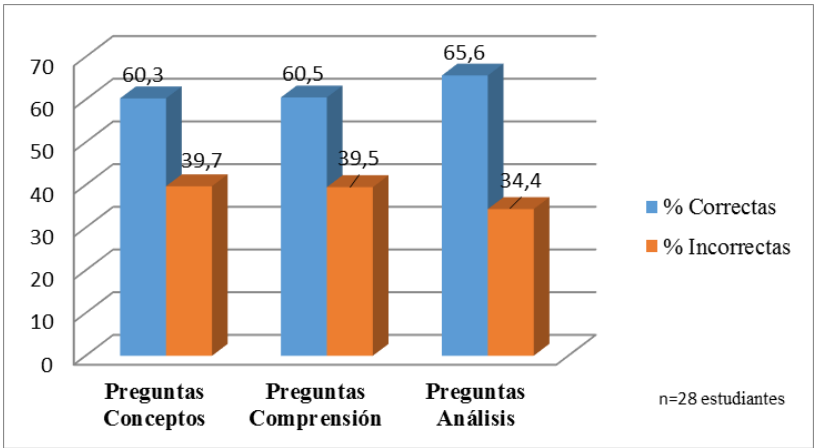

Figura 1. Distribución del porcentaje de respuestas correctas e incorrectas según tipo de preguntas en prueba de conocimientos de clases abordadas con FC

\subsection{Resultados de la comparación de notas de la prueba de conocimientos.}

El promedio de notas de la prueba de conocimiento que evaluaron los resultados de aprendizaje de los estudiantes del año $2014 \quad(n=56)$ versus los estudiantes $2015(n=28)$ a los que se les aplicó $\mathrm{FC}$, fue de 4,1 (E.S=0,7) y 4,3 (E.S=0,8) respectivamente (figura 2). La comparación se realizó con prueba Mann-Whitney y se obtuvo un valor $\mathrm{p}=0,285$.

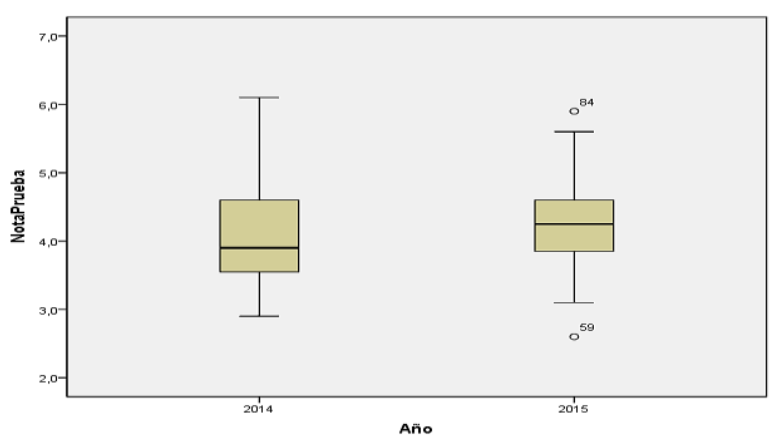

Figura 2. Notas de evaluación de conocimientos de la III Unidad Hematología Clínica. 1-2014 (CT) y 1-2015 (FC).

La distribución de las notas que obtuvieron los estudiantes dependiendo del método de enseñanza se observa en la figura 3.

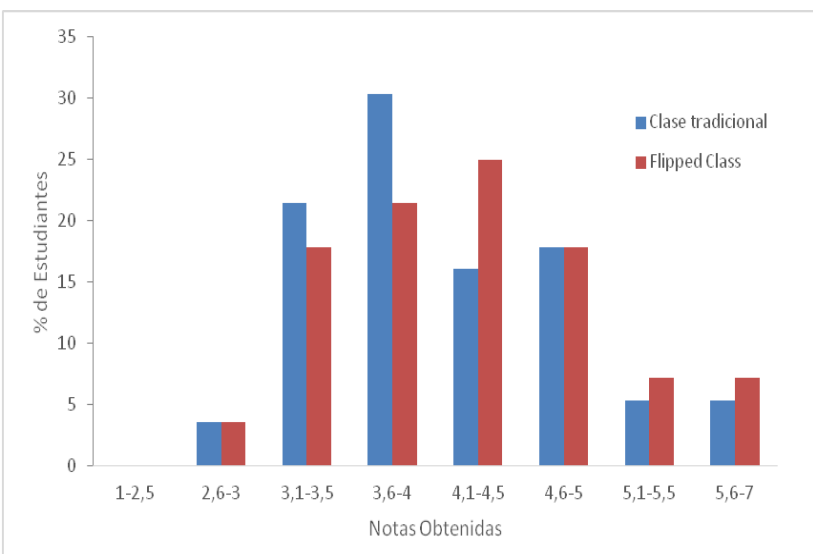

Figura 3. Distribución de notas de prueba de conocimientos obtenidas con metodología de $\mathrm{CT}$ versus $\mathrm{FC}$ 
La comparación de las notas en la prueba de conocimientos de la unidad intervenida, con las del año anterior 1-2015 se realizó con prueba de Chi Cuadrado y se obtuvo un valor $\mathrm{p}=0.043$ (figura 4).

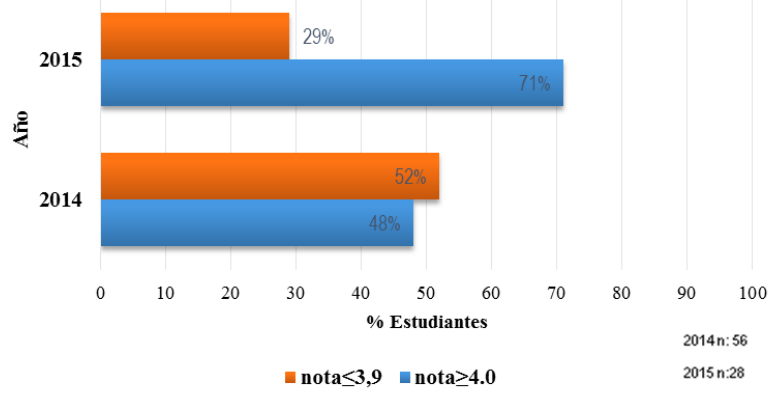

Figura 4. Distribución de notas aprobación versus reprobación en la prueba de conocimientos de la III Unidad

\subsection{Resultado de evaluación de conocimientos y productos durante el desarrollo de la unidad.}

Los resultados de las evaluaciones de conocimiento y producto realizados durante el desarrollo de la unidad de aprendizaje, fueron en promedio de 3,4 $($ E.S $=1,18)$ y 5,5 (E.S=0,51) respectivamente, y se obtuvo un $\mathrm{p}<0.05$ (prueba de Mann-Whitney) -figura $5-$

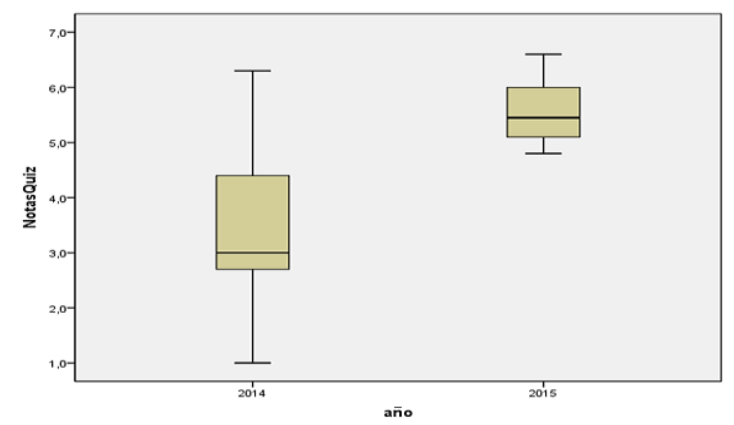

Figura 5. Notas de evaluaciones de producto y conocimiento durante la III unidad

\section{DISCUSIÓN}

Con respecto al ítem 1, el resultado de la encuesta indica que a la mayoría de los estudiantes les gusta ver un vídeo de los contenidos en vez de escuchar la conferencia del docente, aunque hay una minoría que al parecer prefiere la clase tradicional del profesor.

En el ítem 2 (preferir las clases tradicionales del profesor en vez de realizar trabajos activos y grupales en clases como los que se llevaron a cabo con metodología FC) se observa que la mayoría de los estudiantes prefieren las actividades que se realizan con FC en comparación a las que se realizan con clases tradicionales, pero se observó una opinión neutral en un porcentaje considerable de los estudiantes, similares resultados a los reportados en el estudio de Gilboy (2015). Esto podría relacionarse al ítem 1 debido a la presencia de diferentes estilos de aprendizaje presentes en el curso, por lo que algunos estudiantes son más tradicionalistas y de estilo de aprendizaje más pasivo siendo de preferencia la metodología tradicional.

En el ítem 3 que hacía referencia a que si los vídeos les permitían aprender el material de estudio más eficazmente que al hacer lecturas en solitario, la mayoría estaba de acuerdo o muy de acuerdo. En el estudio de Gilboy (2015) se obtuvieron similares respuestas, lo que indicaría, según los estudiantes, que disponer del material audiovisual les ayuda y facilita el aprendizaje porque les permite aprender a su ritmo y repetir el material las veces que sea necesario para lograr el aprendizaje.

El ítem 4 que les preguntaba si ellos habían aprendido más con el método FC en comparación con el método tradicional, la mayoría de los estudiantes estuvieron de acuerdo o muy de acuerdo y ningún estudiante percibe que la metodología invertida no le ayuda a aprender. Esto es explicado por la disponibilidad de revisar el material de estudio según las necesidad de cada estudiante y de la posibilidad de realizar trabajo de aprendizaje activo y colaborativo con los estudiantes en las sesiones presenciales junto con la guía y retroalimentación en tiempo real del docente (Tourón, Santiago y Díez, 2014), junto con que se aplica una enseñanza más individualizada y existe un mejor control del avance de aprendizaje de cada estudiante.

El ítem 5, que les preguntaba si ellos se sentían muy desconectados en las actividades virtuales cuando no tenía la presencia de un profesor, la minoría de los estudiantes responde que están de acuerdo o muy de acuerdo, similar resultado que en el estudio de Gilboy (2015). Por lo que se deja en evidencia que la mayoría de los estudiantes pueden realizar la etapa de adquisición de conocimientos de manera autónoma y a su propio ritmo haciendo uso de las TIC y es más valorado contar con la ayuda y retroalimentación del docente cuando se realizan actividades de mayor complejidad cognitiva (Tourón, Santiago y Díez, 2014) como las que se realizaron en las clases de trabajo activo.

Por lo tanto, de la encuesta de satisfacción aplicada se obtiene que la mayoría de los alumnos perciben alto grado de satisfacción de la metodología FC, aceptan y aprueban ver un vídeo de los contenidos en vez de escuchar la conferencia del 
docente y prefieren el trabajo activo con la guía del profesor durante las sesiones presenciales, lo que otorga gran valor a la metodología debido a que son las respuestas de los propios estudiantes involucrados en el cambio metodológico.

En los ítems de la encuesta se observa un porcentaje de estudiantes que se orientaron por respuestas neutrales (Gilboy, 2015), lo que puede estar influenciado por falta de adherencia hacia la metodología por una parte de estudiantes, por los diferentes estilos de aprendizajes presentes en el grupo de estudio, también a que pueden estar acostumbrados a clases tradicionales impartidas por los docentes y no a metodologías que requieren mayor tiempo de preparación y estudio por parte de los estudiantes y por el cambio que significa responsabilizarse de su propio aprendizaje y participar activamente en las clases construyendo su conocimiento mediante la cooperación y colaboración con otros estudiantes. Adicionalmente a lo anterior, puede influir en que esta es la primera vez que estos estudiantes trabajan con esta metodología de enseñanza aprendizaje y pudiese influir el periodo de adaptación y manejo de la metodología. Además, el grupo de estudio pertenece al cuarto año de formación profesional, por lo que han estado sometidos a metodologías tradicionales en la mayor parte del tiempo de su formación. Sin embargo, estos resultados son similares a otros estudios (Gilboy, 2015; Simpson y Richards, 2015; Moffett, 2014), que mostraron similares niveles de aceptación de la metodología.

Con respecto a los resultados prueba al realizar el análisis con respecto al tipo de preguntas que se incorporaron a la prueba de conocimientos, se obtuvo que prácticamente no hubo diferencias en la adquisición de los aprendizajes tipo I y II, pero las preguntas de mayor complejidad (tipo III) fueron contestadas correctamente en un 5\% más que las otras. Esto podría significar que esta metodología mejora el aprendizaje de los estudiantes relacionados a la aplicación y análisis (aprendizajes de mayor nivel cognitivo según taxonomía de Bloom modificada de Anderson 2001), sin perjudicar los aprendizajes de nivel inferior.

Con respecto a la comparación de las notas de los estudiantes del año 2014 y 2015, que no se haya obtenido diferencias estadísticamente significativas no significa que esta metodología no sirva para el aprendizaje. Podría deberse al bajo número estudiantes de la muestra de este estudio y que corresponden a estudiantes típicamente tradicionales de una cohorte en particular. También podría estar influenciado por las variables intrínsecas (aptitudes cognitivas, estado de salud física y emocional, motivación, interés por los contenidos, disciplina, etc.) y/o extrínsecas de los estudiantes (ambiente sociocultural, condiciones de la sala de clases o de estudio personal, uso eficiente del tiempo, horas de estudio, relación con los compañeros, etc.) que pueden influir en el rendimiento académico y en la percepción de este método y que no es posible controlar. Sin embargo, se observa una tendencia positiva ( $+0,2$ puntos de diferencia) equivalente a un $4,9 \%$ de aumento de las calificaciones.

Muchos docentes y estudiantes argumentan que esta metodología es una herramienta valiosa para incentivar la participación en clases de los estudiantes $y$ en promover el sentido de responsabilidad del mismo en su proceso de aprendizaje de los estudiantes (Gilboy, 2014; O'flaherty, 2015; Simpson y Richards, 2015). Por otro lado, el proceso de adaptación a los cambios metodológicos puede ser lento y podría influir en que no se vean cambios significativos al comienzo de la aplicación de las nuevas metodologías.

Al comprarar las notas obtenidas por los estudiantes con metodología FC y CT, se observó que los estudiantes que normalmente obtienen mejores calificaciones, el uso de esta metodología les permite potenciar su aprendizaje porque se ve que este grupo de estudiantes mejoran más sus calificaciones. No así los estudiantes que obtienen las calificaciones más bajas, en la que no se observa variación dependiendo del método de enseñanza. Se observa que al aplicar FC disminuye el porcentaje de estudiantes que obtienen notas entre 3.1 y 4.0 , lo que podría corresponder a estudiantes que aumentan su rendimiento académico y que, mediante la aplicación de FC, podrían obtener calificaciones superiores.

Por lo tanto, cuando se comprara entre la aprobación y reprobación de la prueba de conocimientos entre los estudiantes del grupo al que se les aplicó metodología FC y al del año anterior, se observa diferencia estadísticamente significativa, es decir, que mediante el usos de la metodología FC se logra obtener mejores resultados de aprendizaje; en este caso, está evidenciado en la nota de la prueba de conocimientos, en la cual la mayoría de los estudiantes logra obtener más de la nota mínima para la aprobación de la evaluación $(71 \%$ en año 2015, versus el $48 \%$ en 2014).

Estos resultados podrían indicar que el aumento de la frecuencia de las notas sobre 4.0 estaría favorecido, por un lado a la mejor preparación de los estudiantes al realizar las actividades previas a las clases y, por otro lado, a las actividades de aprendizaje activo que se realizan en las clases, que permiten involucrar a los estudiantes en la 
construcción de su propio conocimiento. También puede influir la mayor participación y resolución de dudas, especialmente de aquellos estudiantes que normalmente no realizan preguntas y en estas clases más personalizadas y activas pueden responder las inquietudes. De esta manera, se otorgaría mayor flexibilidad a la enseñanza, se optimizaría el tiempo de interacción del docente con el estudiante y se fortalecería el trabajo autónomo de los estudiantes. Por lo que esta metodología de clases invertidas podría favorecer el aumento rendimiento académico de los estudiantes en comparación a las clases tradicionales.

Yestrebsky (2015), en el estudio realizado en la Universidad Central de Florida (EE.UU.), encontró que los estudiantes que obtenían mejores calificaciones (A o B) se veían más beneficiados con la metodología FC y un mayor porcentaje de estudiantes lograban obtener mejor calificación comparados con los que obtenían D o $\mathrm{F}$, en los que no se veía variación en el rendimiento académico. Se observó una leve disminución del porcentaje de estudiantes que obtenían C. En el rendimiento del curso total no se vio mejora en la calificación del curso (-0.3\% disminución de la graduación), pero se vio que mejoró el rendimiento de los estudiantes que normalmente obtienen mejores calificaciones. Similares resultados a los obtenidos en este estudio.

Entre las limitantes de este estudio pusiese estar que los estudiantes son de cohortes diferentes, con número de estudiantes diferente, aunque comparten características de distribución de género, edad y un perfil de ingreso a la carrera similar. Otra limitante es que las clases de realizaron en las salas de la Universidad Santo Tomás que fueron diseñadas para un modelo tradicional de clases y lo ideal es contar con una sala acondicionada para el trabajo activo que cuente con mesas y sillas móviles, equipos audiovisuales y computadores acordes al trabajo activo y colaborativo que necesita esta metodología.

Cuando se compara la evaluación de producto en ambos grupos se observa diferencia estadísticamente significativa entre las notas de los productos evaluados del año 2014 y 2015. Este resultado podría estar influido porque mediante la aplicación de FC se logra que los estudiantes lleguen mejor preparados a la clase, además al considerar el trabajo realizado en clases como parte de la evaluación del curso se sienten motivados para realizar las tareas de aprendizaje activo.

En el estudio de Yestrebsky (2015), en la nota final del curso se ponderó con un $3 \%$ de calificaciones derivadas de la participación de los estudiantes en las clases y actividades virtuales, lo que originó que el rendimiento del curso comparado con el curso de clases tradicionales subiera un $0.7 \%$, lo que revela, al igual que en este caso, que es necesario incluir un ítem de nota relacionado al desarrollo de las actividades previas y de clases como un factor motivacional para fomentar el trabajo por parte de los estudiantes.

\section{CONCLUSIONES}

Es posible realizar una planificación metodológica didáctica para la aplicación de FC, adaptando las actividades de acuerdo a los resultados de aprendizaje de los programas de estudio y aplicando las TIC.

En este estudio se evidenció, al igual que en otros estudios, que los estudiantes prefieren esta metodología en vez de las clases tradicionales. Asimismo, se observó que ellos perciben que aprenden más y mejor con esta dinámica de clases.

En cuanto al rendimiento académico de los estudiantes, se observó que aumenta aproximadamente un 5\%.

Por otro lado, el factor motivacional es un elemento clave para lograr aprendizajes significativos, por lo que con esta metodología se logra crear interés en el aprendizaje activo, haciendo uso de esta nueva forma de transmisión de información y generación de conocimiento y permite generar mayor autonomía en el aprendizaje de los estudiantes y reforzar las habilidades transversales necesarias para desarrollar el profesionalismo en los estudiantes.

Este estudio entrega resultados preliminares debido a que la implementación y evaluación de la metodología se realizó con número limitado de participantes y en un breve periodo de tiempo, pero proporciona un punto de partida para futuras investigaciones.

\section{REFERENCIAS}

Anderson, L., y Krathwohl, D. (2014). Anderson y Krathwohl Revisando la Taxonomía de Bloom. Recuperado a partir de https://eduarea.wordpress.com/2014/11/09/andersony-krathwohl-revisando-la-taxonomia-de-bloom/

Baepler, P, Walker J, Driessen M. (2014). It's not about seat time: blending, flipping, and efficiency in active learning classrooms. Computers y Education, 78, 227 236.

Bergmann, J. (2012). To flip or not to flip? Learning and leading with technology. Recuperado de: http://jonbergmann.com/to-flip-or-not-to-flip/

Berrett, D. (2012). How 'flipping' the classroom can improve the traditional lecture. The chronicle of higher education. Recuperado de: http://www.chronicle.com/article/How-Flipping-theClassroom/130857/

Bishop J., y Verleger M. (2013). The flipped classroom: a survey of the research. American Society for 
Engineering Education. Asee annual conference. Atlanta, GA. Recuperado de: https://www.asee.org/public/conferences/20/papers/6219 /view

Das, Bb, y Sarkar, C. (2015). An innovative flipped class intervention to improve dose calculation skills of fhase I medical students: a preliminary study. Procedia - social and behavioral sciences 182, 67-74.

Fukushi, K. (2010). El nuevo estudiante y el desafío de la meritocracia: análisis del cambio cultural en la educación superior chilena. Calidad de la Educación, 33, 303-316.

Gilboy M. (2015). Enhancing student engagement using the flipped classroom. Journal of nutrition education and behavior. 47(1): 104-114.

Healey, M., y Jenkins, A. (2000). Kolb's experiential learning theory and its application in geography in Higher education. Journal of Geography, 99, 185-195

Hughes, H. (2012). Introduction to Flipping the College Classroom. En T. Amiel y B. Wilson (Eds.), Proceedings of EdMedia: World Conference on Educational Media and Technology 2012 (pp.24342438). Association for the Advancement of Computing in Education (AACE).

Johnson, L., y Renner, R. (2012). Effect of the flipped classroom model on a secondary computer applications course: student and teacher perceptions, questions and student achievement. Graduate thesis, College of Education and Human Development, University of Louisville. EEUU. Recuperado

de https://theflippedclassroom.files.wordpress.com/2012/04 /johnson-renner-2012.pdf

Kachka, P. (2012). Understanding the flipped classroom: part 1. Teaching with technology. Faculty Focus. Higher ed teaching strategies from magna publications. Recuperado http://www.facultyfocus.com/articles/teaching-withtechnology-articles/understanding-the-flippedclassroom-part-2/

Khan Academy (2010). Bill gates talks about the khan academy at aspen ideas festival 2010. Recuperado de https://www.khanacademy.org/talks-andinterviews/keymedia-pieces/v/bill-gates-talks-about-the-khanacademy-at-aspenideas-festival-2010 11 .

Moffett, J. (2014). Evaluation of the flipped classroom approach in a veterinary professional skills course. Advances in Medical Education and Practice, 5, 415-425.

O'flaherty, J. (2015). The use of flipped classrooms in higher education: A scoping review. Internet and Higher Education, 25, 85-95.

Padilla, A. (2007). Tecnologías de información y comunicaciones (TICs) en el aula del siglo XXI: cómo acompañar a profesores y profesoras en este desafío. Pensamiento educativo, 40(1), 391-411.

Phillips, C. (2014). Millennial student and the flipped classroom. Proceedings of ASBBS. 21(1): 519-560.

Phitayakorn, R, et al. (2015). Wise-md usage among millennial medical students. The american journal of surgery, 209, 152-157.

Pozo, J., y Pérez, M. (2011). Psicología del aprendizaje universitario. La formación en competencias". Perfiles educativos XXXIIII, XXXIII, (131). Recuperado de http://www.redalyc.org/articulo.oa?id=13218531013er.

Riveros, X. (2013). Diagnosticando nuestra praxis pedagógica: Definición de tipos de aprendizajes definidos por Universidad Santo Tomás. Vicerrectoría académica. Universidad Santo Tomas.
Simpson, V, y Richards, E. (2015). Flipping the classroom to teach population health: Increasing the relevance. Nurse Education in Practice, 15,

Tourón, J., Santiago, R., y Díez, A. (2014). The Flipped Classroom: Como convertir la escuela en un espacio de aprendizaje. Edición: 1. Digital Text.

Unesco (2005). Hacia las sociedades del conocimiento. Ediciones UNESCO Recuperado de: http://unesdoc.unesco.org/images/0014/001419/141908s .pdf.

Unesco (2006). Las tecnologías de la información y la comunicación en la enseñanza. Ediciones UNESCO. Recuperado http://unesdoc.unesco.org/images/0013/001390/139028s .pdf.

Whillier, S., y Lystad, P. (2015). No differences in grades or level of satisfaction in a flipped classroom for neuroanatomy. The journal of chiropractic education, 29(2), 127-133.

Yarbro J. (2014). George Mason University. Extension of a Review of Flipped Learning. Recuperado de: http://flippedlearning.org/wpcontent/uploads/2016/07/Extension-of-FLippedLearning-LIt-Review-June-2014.pdf

Yestrebsky C. (2015). Flipping the Classroom in a Large Chemistry Class-research University Enviroment. Procedia - Social and Behavioral Sciences. 191(2), 1113-1118.

Zappe, S., Leicht, R. Messner J., Litzinger, T., Woo Lee H. (2009). 'Flipping' the classroom to explore active learning in a large undergraduate course. American Society for Engineering Education. Recuperado de: https://peer.asee.org/flipping-the-classroom-to-exploreactive-learning-in-a-large-undergraduate-course.pdf. 